\title{
COMMENTAIRES ET DISCUSSIONS
}

\author{
HAUTEUR D'EAU A L'EXTRÉMITÉ D'UN LONG DÉVERSOIR \\ par Ch. JAEGER, \\ English Electric Company Rugby
}

M. A. CRAYA a mentionné (r) ici même, un résultat trouvé expérimentalement par HUNTER ROUSE $(2)$ : à l'extrémité d'un déversoir à très large seuil (type de Bélanger) la hauteur d'eau $h_{\text {. }}$ est une fraction de la hauteur critique $h_{\mathrm{c}} \stackrel{\sim}{=} \frac{2}{3}$ H. M. H. ROUSE a constaté que (voir fig. 1).:

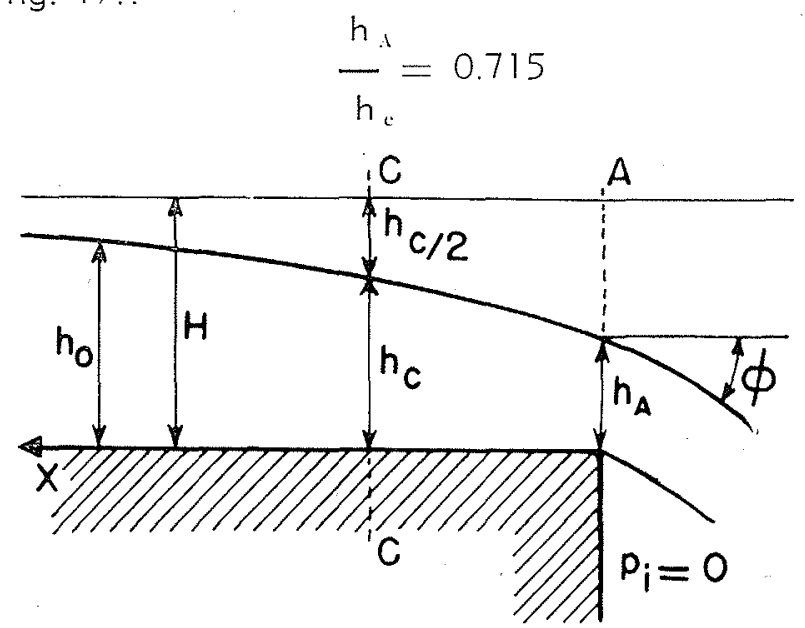

A

Fig. 1

D'après $M$. CRAYA, le théorème de BERNOUILLI donne:

$$
\frac{h_{i}}{h_{c}}=0.65
$$

Pour corriger ce résultat, $M$. CRAYA indique qu'il faudrait, en outre, tenir compte de l'inclinaison des filets et $d^{\prime}$ une petite pression rési.. duelle. A première vue, c'est un problème d'intérêt limité. Placé dans le cadre convenable, il prend plus d'ampleur.

(1) A. CRAYA - "Lo Houille Blanche " - Mars crril 1948 - page 185

(2) H. ROUSE - Fluid Mechanics for hydraulic Engineers - page 325 .
Le problème soulevé par les essais de H. ROUSE nous avait occupés il y a quelques années déjà. De façon plus générale, nous nous étions de. mandé quelles relations lient le théorème de BERNOUILLI et l'équation des quantités de mouvement dans un profil en travers quelconque, et nous cherchions l'existence possible d'un théorème d'extremum. Pour répondre à nos questions, aucun problème particulier n'était sans intérêt.

Tout d'abord, le cas du déversoir à crête arron. die nous parut à ce sujet, digne d'attention (3). C. FAWER ( +1 avait montré, en suivant une méthode de tâtonnement et en utilisant les procédés graphiques de PRASIL, que le débit d'ur déversoir à crête arrondie était un débit maximum pour une charge donnée $H$. Des essais; puis le colcul direct permirent de contrôler $\mathrm{c}$ résultat et de prouver, en outre, que en même temps et au même point, la charge $H$ était minimum pour un débit $Q$ donné (5). Cette coïncidence prêtait à réflexion. Elle n'était d'ailleurs nullement fortuite, le déversoir à crête arrondie étant un cas particulier du théorème d'extremum cherché. Nous avons pu le montrer ailleurs (s)

Le cas du déversoir à large crête permet d'étu. dier un autre aspect du même groupe de problèmes. Ici, sans hésitation aucune, on peut appliquer simultanément le théorème de BELANGER du débit maximum $\frac{\delta Q}{\delta h}=0$ pour $H=$ const) et celui de BOESS donnant le mi-

(3) CH. JAEGER - Notes sur le calcul des déversoirs et seuils. "Bulletin Technique de la Suisse Romande » 24 juin et 8 juillet 1933.

(4) C. FAWER - Etude de quelques écoulements ì filets courbes - Lousanne - 1937.

(5) CH. JAEGER - Contribution à l'étude des courants liquides à surface libre. Revue Générale de l'Hydraulique - $n^{\circ} 33-34$ - 1943 - et Bulletin Technique Suisse Romande -7 et 31 Août 1943 . 
nimum de la charge ou ligne d'énergie $\left(\frac{\delta H}{\delta \mathrm{h}}=\right.$ o pour $\mathrm{Q}=$ const ${ }^{-=}$La courbure des filets liquides est négligeable dans la section critique, où $h=h_{c}$. Tout cela est bien connu.

Mais on peut se demander quelles sont les relations entre l'énergie et les quantités de mouvement entre les profils $A-A$ et $C$-C. C'est le problème que NEBBIA $\left(^{6}\right)$ a posé de façon géné.rale et qu'il a cherché à résoudre, mais en négli.. geant la courbure des filets et l'inégale répartition des vitesses, ce qui fausse, en certains cas, ses conclusions. Les mesures de H. ROUSE (7) donnent, pour le déversoir à large crête, une réponse à cette question: Les deux théorèmes de l'énergie (BERNOUILLI) et des quantités de mouvement (BRESSE) sont valables entre $A A$ of CC. On peut donc songer à faire usage simultanément des deux théorèmes pour retrouver la forme de la ligne d'eau et analyser la répartition des vitesses et des pressions à l'intérieur de la masse liquide.

L'idée d'appliquer simultanément les deux théorèmes de l'hydraulique est probablement nouvelle. Voici ce que donne le calcul. Dans le

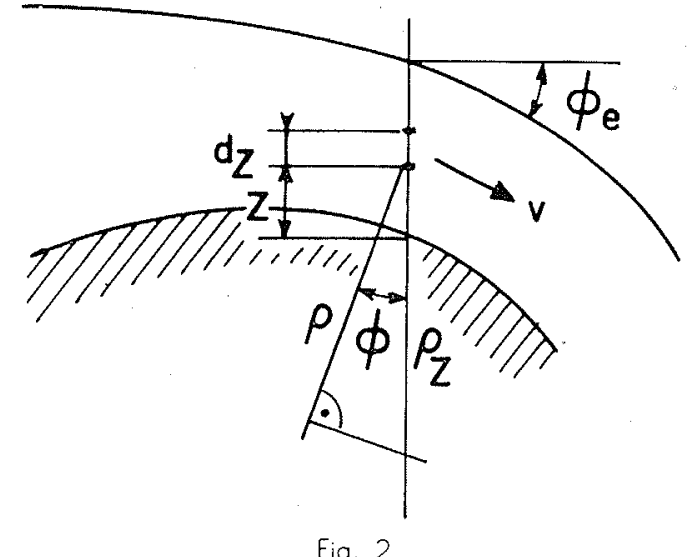

cas des filets incurvés (fig. 2) I'on peut écrire, en première approximation :

$$
\frac{d v}{v}+\frac{\cos \phi}{p} d z=0
$$

Si l'écoulement s'effectue sur radier plan, on écrira avec avantage que le rayon de courbure

(6) G. NEBBIA - Linee d'energia, profili e linee delle quontitò di moto in correnti liquide permonente gradual.. mente varie - Acqua e Gas, Février 1938.

(7) H. ROUSE - Verteilung der Hydraulischen Ener * jie bei einem lotrechten Absturz - Karlsruhe - 1933. varie de façon continue avec $Z$ d'après la loi simple :

$$
\frac{1}{\rho_{Z}}=\frac{\cos \phi}{\rho}=\frac{1}{R_{e}}\left(\frac{z}{h}\right)^{n}
$$

où $R_{c} \cos \varphi_{\text {e }}$ est le rayon de courbure en sur-face, $n$ un paramètre qu'on déterminera de cas en cas. C'est d'ailleurs la seule hypothèse que nous introduisons dans le calcul.

La fonction $\rho_{\%}$ satisfait aux conditions, aux limites.

En effet, pour :

$$
\begin{array}{ll}
Z=0 & \rho_{0}=\infty \\
Z=h & P_{Z \times h}=R_{0}
\end{array}
$$

Introduisons $\rho_{\%}$ dans l'équation (1); il vient :

$$
\frac{d v}{v}=-\frac{z^{n}}{R_{e} h^{n}} d z
$$

Posons que, d'après BERNOUILLI:

$$
\begin{aligned}
& z=n, v=v_{0}=\sqrt{2 g(H-n)} \\
& z=0, v=v_{i}=\sqrt{2 g\left(H-p_{i} / \gamma\right)}
\end{aligned}
$$

(signification des indices: $i=$ intérieur $\mathrm{e}=$ extérieur).

Nous pouvons alors intégrer et transformer (3) ; pour $n \neq-1$, on $a$ :

$$
\ln \frac{v_{e}}{v}=-\frac{1}{R_{e} h^{n}} Z_{z}^{n} d z=-\frac{1}{(n+1) R_{e} h^{n}}\left(n^{n+1}-Z^{n+1}\right)
$$

et

$$
v=v_{e} e^{\frac{1}{(n+1) R_{e} h^{n}}\left(h^{n+1}-z^{n+1}\right)}
$$

ou encore :

$$
v=v_{e} \quad e^{k}\left(1-\frac{z^{n+1}}{h^{n+1}}\right)
$$

avec

$$
K=\frac{h}{(n+1) R_{c}} \quad v_{e}=\sqrt{2 g(H-h)}
$$


$\checkmark$ est ici une fonction de $H$, h et $Z$ d'une part d'autre part de $R_{e}$ et $n$. $R_{\text {, et }} n$ dé. pendent des conditions aux limites particulières du problème. Connaissant $v$ et $v$. on en déduit la vitesse $v$ et la pression $P$ en un point quelconque de la section A-A.

Pour

$$
z=0, \quad v=v_{i}=v_{e} e^{h /(n+1) R} e=v_{e} e^{K} \quad \text { et }
$$

ou encore:

$$
\frac{v_{e}}{v_{i}}=e^{-k}=\sqrt{\frac{H-h}{H-p_{i} / Y}}
$$

$$
\left.\frac{p_{i}}{y}=H-e^{2 K}(H-h)\right)
$$

Appliquons ces équations au problème parti. culier qui nous occupe.

Dans ce cas, au point $A$ on peut poser $p_{i}=0$ (pression atmosphérique).

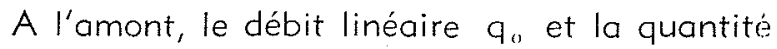
totale de mouvement (BRESSE) $S=\frac{h_{0}{ }^{2}}{2}+$ $\frac{v_{\mathrm{o}^{2}}}{\mathrm{~g}} h_{0}$ sont donnés. (Le poids spécifique $\gamma$ de l'eau est égal à l'unité). Le débit doit satisfaire à l'équation.

$$
\begin{aligned}
q=\int_{0}^{h} v d z & =v_{e} \int_{0}^{h} e^{k\left[1-\left(\frac{z}{h}\right)^{n+1}\right]} d z \\
& =v_{e} e^{k} e_{0}^{-k\left(\frac{z}{h}\right)^{n+1}} d z
\end{aligned}
$$

Posons :

$$
\begin{aligned}
& \omega=-k\left(\frac{z}{h}\right)^{n+1} \\
& d \omega=-K \frac{n+1}{h^{n+1}} z^{n} d z=-k^{1 / n+1} \frac{n+1}{h}(-\omega)^{n / n+1} d z
\end{aligned}
$$

On en tire :

$$
q=v_{e} e^{k} \frac{(-h)}{(n+1) k^{1 / n+1}} \int_{0}^{-k} \frac{e^{w}}{(-w)^{n / n+1}} d w
$$

qui est de forme connue.
L'équation des quantités de mouvement peut être mise sous la forme

$$
\int\left(\frac{v^{2}}{g}+\frac{p}{y}\right) d z
$$

Or, pour chaque filet liquide :

$$
H=z+\frac{p}{\gamma}+\frac{v^{2}}{2 g}
$$

Si f'on néglige les pertes par turbulence et si l'on admet un écoulement potentiel, $H$ est une constante pour toute la section et l'on peut écrire:

$$
\int=\int_{0}^{h}\left(H-z+\frac{v^{2}}{2 g}\right) d z=H h-\frac{h^{2}}{2}+\frac{1}{2 g} \int_{0}^{h} d z
$$

ou encore :

$$
\int=H h-\frac{h^{2}}{2}+\frac{v_{e}^{2}}{2 g} e^{2 k} e_{0}^{-2 k\left(\frac{z}{h}\right)^{n+1}} d z
$$

lci encore, on posera:

$$
\begin{aligned}
u & =-2 k\left(\frac{z}{h}\right)^{n+1} \\
d u & =-2 k \frac{n+1}{h^{n+1}} z^{n} d z
\end{aligned}
$$

et

$$
\begin{aligned}
\int_{0}^{h} e^{-2 k(z / h)^{n+1}} d z \\
=-\frac{h}{(n+1)(2 k)^{1 / n+1}} \int_{0}^{-2 k} \frac{e^{u}}{(-u)^{n / n+1}} d u
\end{aligned}
$$

On vérifie que la solution:

$$
h_{A}=048 H, \quad n=-1 / 2
$$

convient simultanément aux deux équations 7 ef 8 ou (7a) et (8a). On peut estimer, en première approximation, la valeur $h_{a}$ en utilisant une méthode approchée que nous exposerons plus loin.

\section{COMPARAISON AVEC LES MESURES DE H. ROUSE.}

Comparons maintenant les résultats de calcul 
aux valeurs mesurées par H. ROUSE, Cet auteur a mesuré par unité de largeur $(B=1 \mathrm{~cm}$.) :

$q=1250 \mathrm{~cm}^{3} / \mathrm{sec} . \mathrm{cm}$.

$\mathrm{h}_{\mathrm{i}}=11.9 \mathrm{~cm}$.

$V_{\mathrm{m}}=\mathrm{q} / \mathrm{h}=105 \mathrm{~cm} / \mathrm{sec}$.

$\mathrm{S}=204.5 \mathrm{~cm}^{3} / \mathrm{cm}$.

$H=H_{A}=17.38 \mathrm{~cm}$. (mesuré dans le profil A-A) ;

$$
h_{C} \approx \frac{2}{3} H_{A}=1158
$$

De ces données, nous tirons:

$$
\begin{aligned}
& h_{A}=0.48 H_{1}=0.48 \times 17.38=8.42 \mathrm{~cm} . \\
& h_{A} \\
& h_{\mathrm{c}} \\
& v_{c}=\sqrt{2 \mathrm{~g}\left(\overline{\left.H_{A}-h_{A}\right)}\right.}=133 \mathrm{~cm} . / \mathrm{sec} . \\
& \text { (mesuré } 134 \mathrm{~cm} / \mathrm{sec} \text {.) } \\
& v_{i}=v_{i} / \sqrt{0.52}=185 \mathrm{~cm} / \mathrm{sec} \text {. } \\
& \text { (mesuré } 162 \mathrm{~cm} / \mathrm{sec} \text {.) }
\end{aligned}
$$$$
-K=\ln \frac{v_{\theta}}{v_{1}}=\ln \sqrt{\frac{H_{A}-h_{A}}{h_{A}}}=\ln \sqrt{0.52}=-0.327
$$$$
K=0.327=h_{A} /(n+1) R_{B}=8.42 /(n+1) R_{B}
$$$$
e^{K}=e^{0.327}=1.385
$$$$
\int_{0}^{h} e^{-k(z / h)^{1 / 2}} d z=6.83
$$$$
\int_{0}^{h} e^{-2 k(z / h)^{1 / 2}} d z=5.63
$$$$
q=v_{0} e^{k} \int_{0}^{h} e^{-k(z / h)} d z=1260 \mathrm{~cm}^{3} / \mathrm{sec} c \mathrm{~m}
$$

(mesuré $1250 \mathrm{~cm}^{3} / \mathrm{sec} . \mathrm{cm}$.)

$$
\int e^{-2 K(z / h)^{1 / 2}} d z=\frac{2 g}{v_{B}^{2} e^{2 K}}\left[S_{A}-h_{A}\left(H_{A}-\frac{h_{A}}{2}\right)\right]=5.42
$$

contre 5.63 trouvé en calculant l'intégrale. Les valeurs $h_{A}=0.48 H_{A}$ et $n=1 / 2$ satisfont donc bien simultanément à l'équation de BER.
NOUILLI, à l'équation des quantités de mouvement et à la condition du débit.

En poursuivant les calculs, on trouve pour $n=-1 / 2$

pour $Z=0 \quad R_{i}=0$

pour $z=h_{A} \quad R_{\mathrm{c}}=\frac{h_{\mathrm{A}}}{(\mathrm{n}+1) \mathrm{K}}=51.5 \mathrm{~cm}$.

Cette dernière valeur coïncide de façon satisfaisante avec le profil en lorig relevé par HUN. TER ROUSE. La solution $\mathrm{R}_{\mathrm{i}}=0$ nous parait moins satisfaisante. On sait combien il est difficile d'analyser un écoulement aux environs d'une discontinuité !

Nous avons encore cherché à calculer les coefficients $\alpha_{1} \alpha^{\prime} \beta$, et $\beta^{\prime}$, caractéristiques de l'écoulement et à les comparer aux valeurs qu'on peut déduire du travail de HUNTER ROUSE. Nous avons obtenu:

$$
\begin{aligned}
& a=\frac{h^{2}}{q^{3}} \int_{0}^{h} v^{3} d z=1.066 \text { (calcul) contre }{ }_{\text {(mesure) }}^{1.065} \\
& a^{\prime}=\frac{h}{q^{2}} v_{0}^{2} d z=1.033 \text { (calcul) contre } \begin{array}{c}
1.042 \\
\text { (mesure) }
\end{array} \\
& \beta=\frac{1}{q h} \int_{0}^{h} \frac{p}{\gamma}+z \text { ) } v d z=0.646 \text { (calcul) contre } 0.69 \\
& \begin{array}{l}
\text { (mesure) } \\
v^{\prime}
\end{array} \int_{0}^{h} \frac{p}{\gamma} d z=0.443 \text { (colcul) contre } 0.376 \\
& \text { (mesure) }
\end{aligned}
$$

La concordance entre calcul et mesures nous parait assez satisfaisante, d'autant plus que l'examen des diagrammes des vitesses relevées par $H$. ROUSE prouve qu'il y a, en nature, une légère turbulence aux environs du point $Z=0$. La méthode de calcul proposée permet de saisir correctement non seulement la hauteur $h_{\mathrm{i}}$ mais encore la répartition des vitesses et des pressions. A notre point de vue, c'est là le résultat essen. tiel du calcul et de la discussion soulevée par $M$. CRAYA. Il est donc possible, en combinant les notions d'énergie et d'impulsion, de décrire de façon convenable, ce qui se passe à l'intérieur d'un écoulement.

Reste à déterminer la tangente à la ligne d'eau dans la section A-A. Pour cette dernière estimation, nous allons avoir recours à la méthode de calcul approchée de C. FAWER. Sans 
insister sur le détail de cette méthode, nous dirons simplement qu'elle revient à négliger l'inégale répartition des vitesses dans le profil en travers, c'est-à-dire à poser $\alpha \cong$ I et à poser en outre, pour le rayon de courbure en surface $1 / R_{e} \stackrel{\sim}{=} d^{\prime \prime} h / d x^{2}$ Ces mêmes hypothèses restric. tives sont à la base du calcul des pressions. Tel est le sens de la méthode. Elle ne permettrait ni le calcul de la répartition des vitesses, ni même la détermination de $h_{A}$ et de $n$. Ces valeurs, une fois calculées, ainsi que nous l'avons fait plus haut, la méthode de FAWER est, par contre d'un emploi très utile pour nous orienter. sur l'inclinaison des filets liquides dans le profil A-A.

Reproduisons l'équation de FAWER:

$$
\frac{1}{2}\left[\left(\frac{d h}{d x}\right)^{2}-\operatorname{tg}^{2} \phi_{0}\right]=\frac{n+2}{h_{c}^{3}}\left[H \frac{h^{2}-h_{0}^{2}}{2}-\frac{h^{3}-h_{0}^{3}}{3}-\frac{h_{c}^{3}}{2} \ln \frac{h}{h}\right]
$$

$h$ et $\varphi$ étant la valeur de $h$ et de l'angle en surface dans un profil initial quelconque.

Nous nous heurtons, ici, à une imperfection de la méthode (8) qui ${ }^{(8}$ 'introduit pas les dérivées par rapport à l'abscisse $X$; il faut donc choisir arbitrairement comme point de départ, un point de la courbe relevée par $H$. ROUSE. Pour le point situé à $155 \mathrm{~cm}$. à l'amont de la section $\mathrm{A}-\mathrm{A}$, on trouve :

$$
\begin{aligned}
& h_{0}=13.56 \mathrm{~cm}, \operatorname{tg} \phi_{0}=0.032 \text { ef } \mathrm{tg}^{2} \phi_{0}=0,001 \\
& \text { Or } q=1250 \mathrm{~cm}^{3} / \mathrm{sec} \text { et } h_{c}=\sqrt{\frac{q^{2}}{g}}=11.60 \mathrm{~cm}
\end{aligned}
$$

donc:

$$
\begin{gathered}
\lg y=\frac{d h}{d x}=0.221 \\
\phi=12^{\circ} .30^{\prime}
\end{gathered}
$$

dans la section A-A.

La valeur mesurée par HUNTER ROUSE est $\varphi=14^{\circ} 30^{\prime}$. La concordance pourrait être

(8) Nous avons introduit les dérivées par rapport i l'abscisse dans une étude parue dans la Revue Générale de I'Hydraulique $n^{\circ} 37$ à 41 - 1947 -. La forme exacte de la ligne d'eau dépend ici du coefficient de rugosité du lit et, en pratique, on se servira du théorème de BERNOUILLI. meilleure. Vraisemblablement, ainsi que nous l'avons déjà dit, les pertes diverses, frottements et turbulence jouent un certain rôle dans le profil A-A, et à l'amont de ce profil.

\section{METHODE APPROCHEE POUR LE CALCUL DE $h_{A}$}

La méthode suivante donne rapidement une valeur approchée de $h_{\text {.1. }}$.

Considérons la masse d'eau qui, pendant l'espace de temps $d t$, franchit la section A-A avec une vitesse movenne $V_{n}=\mathrm{q} / \mathrm{h}$. . Le centre de gravité de cette masse d'eau se trouve environ à la hauteur hi/2 au-dessus du seuil. Sur une courte distance, nous pouvons confondre la trajectoire de ce centre de gravité avec une parabole à axe vertical et.tangente horizon. tale au sommet, le sommet étant placé au centre de gravité de la section A-A. Cette parabole a pour équation :

$$
y=\frac{1}{2} g t^{2}=\frac{1}{2} \frac{g}{v_{m}^{2}} x^{2}=\frac{g h_{A}^{2}}{2 q^{2}} x^{2}
$$

(le système d'axes passe par le centre de gravité de la section A-A, I'axe $x$ est horizontal)

Pour intégrer aisément l'équation (1) nous admettons que:

$$
\rho_{z}=\frac{\rho}{\cos \phi} \widetilde{x} R_{i}+2
$$

On aura donc pour courbure du filet liquide moyen :

$$
\begin{aligned}
& \frac{1}{\rho_{m}} \cong \frac{d^{2} y}{d x^{2}}=g \frac{h_{A}^{2}}{q^{2}} \\
& \rho_{m}=R_{i}+\frac{h_{A}}{2}=\frac{q^{2}}{g h_{A}^{2}}
\end{aligned}
$$

D'autre part,

$$
\frac{v_{e}}{v_{1}}=\sqrt{\frac{H-h_{A}}{H-p_{i} / \gamma}}=\frac{R_{i}}{R_{i}+h_{A}}=\frac{q^{2} / g h_{A}^{2}-h_{A} / 2}{q^{2} / g h_{A}^{2}+h_{A} / 2}
$$


Pour $H=17.38 \mathrm{~cm} ., q=1250 \mathrm{~cm}^{3} / \mathrm{sec}$. $\mathrm{cm}$, on trouve:

$$
h_{A}=8 \mathrm{~cm}=0.46 \mathrm{H} \text { contre } 0.48 \mathrm{H}
$$

d'après la solution donnée plus haut. II est très utile de connaître approximativement $h_{k}$ avant d'aborder le calcul plus exact.

\section{CONCLUSION}

Les essais de HUNTER ROUSE épuisent, au point de vue pratique, le sujet. Ils nous renseignent parfaitement sur le déversement à l'extrémité d'un déversoir à large crête, et la loi de similitude permet d'extrapoler les résultats trouvés à n'importe quel déversoir similaire.

Du point de vue théorique, l'intérêt est moins de trouver la hauteur exacte à l'aval du déver.. soir, que de montrer par un exemple précis, comment l'énergie du courant et la quantité de mouvement son't liées à la forme de la ligne d'eau et à la répartition des vitesses et des pressions.

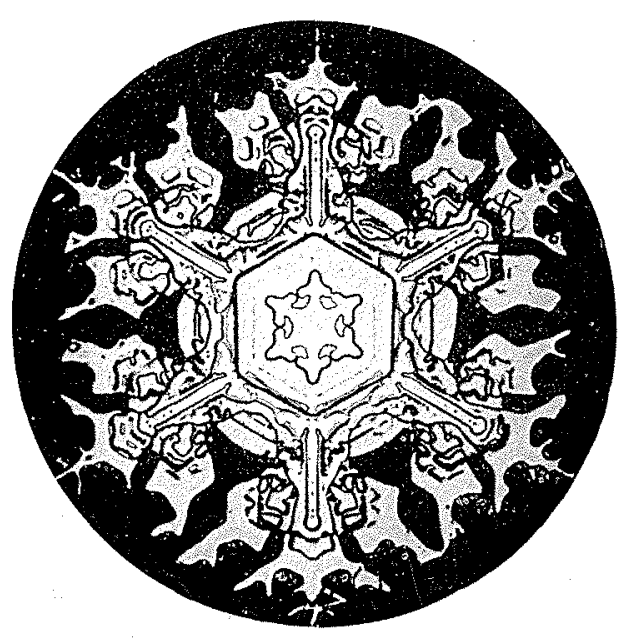

